\title{
Proposição para abordagem algorítmica de planejamento integrado de transportes e uso do solo'
}

\section{Proposition of land use transport integrated planning algorithmic approach}

\author{
Geaquinto, Pedro Dias ${ }^{1}$ \\ 1 Programa de Engenharia de Transportes, Instituto Alberto Luiz Coimbra de \\ Pós-Graduação e Pesquisa em Engenharia, Universidade Federal do Rio de \\ Janeiro, Rio de Janeiro, RJ, Brasil, 21941-914-geaquinto@pet.coppe.ufrj.br
}

\begin{abstract}
RESUMO
A inclusão da integração com o uso do solo acrescenta complexidade aos já difíceis problemas de desenho de rede de transportes. Então, a limitação da cognição humana reivindica o uso de recursos computacionais. Ao mesmo tempo, esses são problemas complexos de se modelar. Esse trabalho se refere a uma exploração metodológica inicial no curso de doutorado em Engenharia de Transportes. O objetivo desse ensaio é estabelecer etapas metodológicas, e suas relações, para compor um método generativo e algorítmico que reconheça a interação entre transportes e uso do solo. Para tanto, foram explorados conceitos da morfologia urbana e da pesquisa operacional, de maneira teórico-conceitual. Como resultado, foram estabelecidas as bases ontológicas de um sistema dinâmico que será desenvolvido ao longo da confecção da tese. Dessa forma, o trabalho desenvolve um método generativo orientado aos objetivos de planejamento em vez das heurísticas restritas por gramáticas, presentes na literatura.
\end{abstract}

Palavras-chave: LUTI, Desenho generativo, Morfologia urbana

\begin{abstract}
The inclusion of integration with land use adds complexity to the already difficult problems of transport network design. Therefore, the limitation of human cognition claims the adoption of computational resources. Meanwhile, these are complex model problems. This paper refers to an initial methodological evaluation within PhD studies in Transport Engineering. The objective of this essay is to establish methodological steps, and their relationships, to compose a generative and algorithmic method that recognizes the interaction between transport and land use. For this purpose, concepts of urban morphology and operational research were explored in a theoretical assessment. As a result, this work establishes the ontological basis of a dynamic system to be developed in the thesis. Hence, this work develops a generative method
\end{abstract}

\footnotetext{
${ }^{1}$ GEAQUINTO, Pedro Dias. Proposição para abordagem algorítmica de planejamento integrado de transportes e uso do solo. In: II SIMPÓSIO NACIONAL DE GESTÃO E ENGENHARIA URBANA: SINGEURB, 2019, São Paulo. Anais... Porto Alegre: ANTAC, 2019.
} 
oriented to the planning objectives instead of heuristics restricted by grammar, as seen in the literature.

Keywords: Land use transport interaction, Generative design, Urban morphology

\section{INTRODUÇÃO}

Nas abordagens que integram transportes e uso do solo, a consistência no uso de fundamentação tipológica materializa os aperfeiçoamentos mais recentes do Desenvolvimento Orientado pelo Transporte Coletivo (KAMRUZZAMAN et al., 2014; LYU; BERTOLINI; PFEFFER, 2016; ZEMP et al., 2011 ). Desde a inserção crescente de dimensões como instrumentos, como interação com o mercado imobiliário e mudanças de uso do solo, o desafio de elencar vocações para tipologias e coordenar territórios com intenção de gerar soluções eficazes se torna árduo para a cognição humana. Assim, o auxílio computacional torna-se essencial para gerar estratégias com impacto esperado das políticas públicas.

Este ensaio objetiva demonstrar capacidades de métodos generativos às técnicas de desenho nesse planejamento integrado. Propõe-se segmentação em etapas definidas, que signifiquem funções simultaneamente distintas e sequenciais e estruturem um método que reúna sofisticação empírica e reconhecimento de processos urbanos.

O trabalho é composto por três partes, fora introdução. A próxima seção volta-se à revisão de implicações de soluções analíticas no planejamento integrado. A seguinte propõe segmentação do planejamento em procedimentos particulares, por exploração metodológica. Finalmente, considerações finais.

\section{ARGUMENTO}

A interpretação do contexto urbano em variáveis quantitativas implica numa abstração paramétrica. Isso permite considerar que algumas dimensões se correlacionam e se recomponham coordenadamente (LIMA; KÓS, 2014). Quando se reconhece que o ambiente construído e sua disposição espacial organizam-se sistematicamente em padrões - regras e relações -, é razoável pressupor que decisões estruturadas por esse tipo de análise possam ser igualmente sistêmicas. Lima $(2017$, p. 86) afirma que a modelagem paramétrica demanda habilidades para conceber fluxos de dados, pensar abstratamente, matematicamente e algoritmicamente. Enquanto os primeiros requisitos são naturais em estudos respectivamente empíricos; teórico-conceituais; e quantitativos, o último predispõe maneira metódica na abordagem: adoção de pensamento abstrato, associativo e hierárquico para decompor soluções em instruções simples (ibid., p.84).

Considera-se, então, que algoritmos podem significar duas posturas no desenho: aquela meramente potencializada por ferramentas avançadas, que deliberem e restrinjam sofisticadamente, e aquela que introduz lógica algorítmica nos próprios processos internos do exercício criativo (PICON, 2006). Essa abordagem torna-se relevante quando o sistema é cada vez mais detalhado e menos compreensível para a cognição humana, porque a concepção desses códigos traduz $\bigcirc$ problema de forma compatível para aplicar linguagens computacionais.

A assistência dessa lógica ao processo criativo permite orientação aos objetivos planejados e concepção de soluções completamente analíticas. Nesse caso, a abordagem se torna equivalente a sistemas generativos, que ultrapassam o motivo estético ao inserir iterações de modelagem e análise de desempenho na própria organização, por simulação ou otimização (LIMA; KÓS, 2014; LIMA, 2017, p. 121).

O que diferencia sistemas generativos do processo tradicional é a substituição da interação direta com o produto pela indireta, com mecanismos que o gerem (FISCHER; HERR, 2001). No urbanismo, aplicações auxiliadas por capacidade computacional são crescentes, embora não tão populares ou desenvolvidas como na arquitetura (LIMA, 2017, p. 26). 


\section{METODOLOGIA}

Quando fundamentado em sequência algorítmica, o planejamento se fraciona em sua estrutura e se configura em etapas seriadas e hierárquicas (LIMA, 2017, p. 84). Essa seção demonstra essas partições e propõe organização na resposta ao problema em três estágios.

Gauthier \& Gilliland (2006) classificam métodos de morfologia urbana conforme duas distinções. A primeira é definida pelas finalidades primárias: contribuições com capacidades "cognitivas", que explicam descrições e processos; contra aquelas com capacidades "normativas", que articulam visões de futuro e ações. A segunda discerne conjunturas epistêmicas conferidas à forma urbana: se a abordagem é "internalista", os atributos estudados consideram-se um sistema independente; se é "externalista", a forma é passiva a processos exógenos. Estudos se inserem num espectro formado por essas dimensões, ilustrado pela Figura 1.

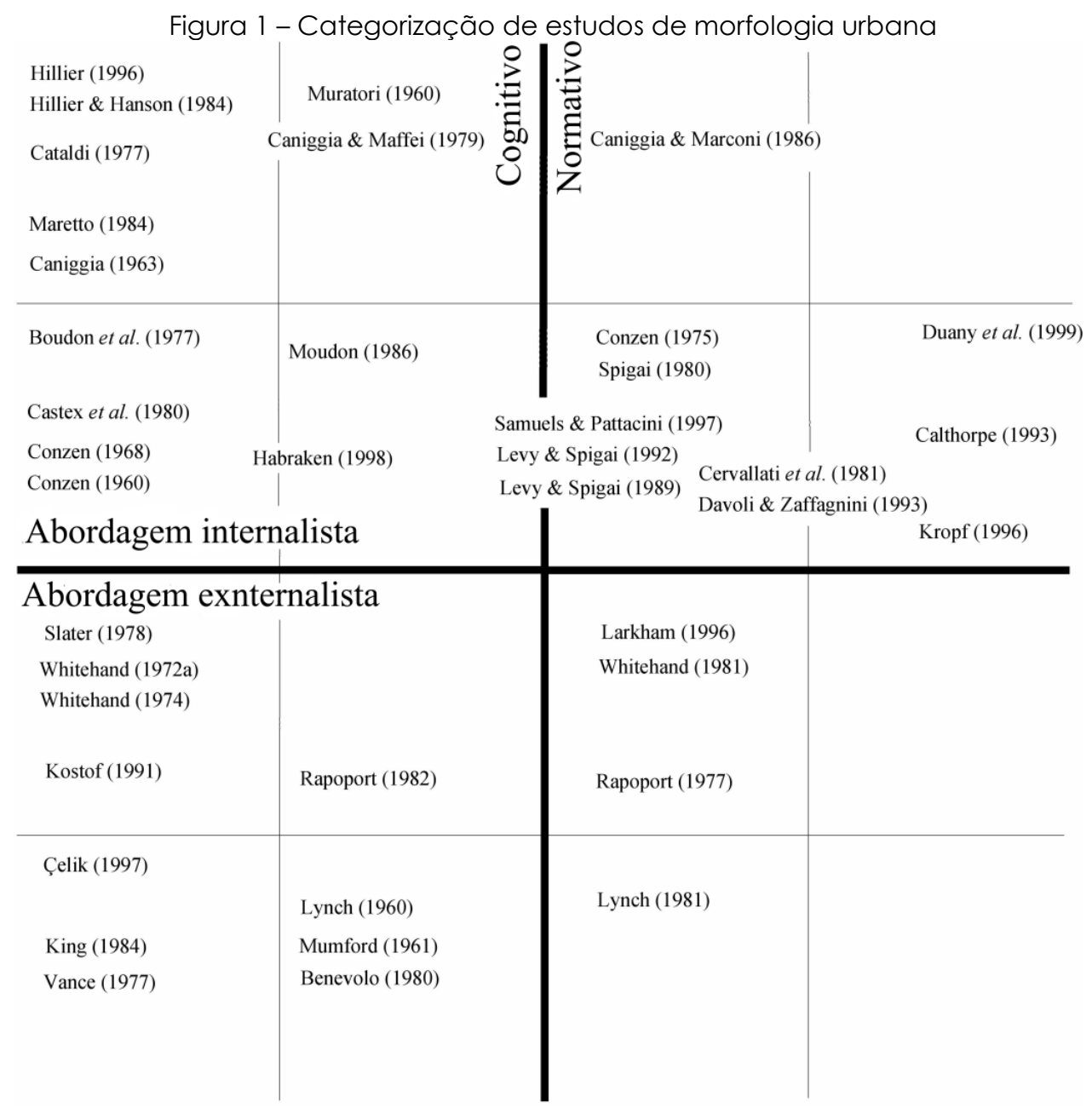

Fonte: Gauthier e Gilliland (2006)

Mera classificação morfológica, entretanto, não admite estratégias sequenciais: a complementaridade entre procedimentos para impactar estratégias não é comentada. Em contrapartida, desenhos generativos são direcionados ao exercício criativo, como o City Induction (DUARTE et al., 2012), subdividido em três submodelos: "formulação", que traduz o contexto em especificações e ingredientes de um plano; "geração", que cria soluções genéricas que são progressivamente moldadas pelo processo de elaboração, conforme uma gramática; "avaliação", que compara soluções perante objetivos teóricos. Portanto, enquanto soluções são restringidas por condições da formulação e são concebidas de acordo com a coesão da geração, suas implicações são ponderadas pela avaliação.

O confronto entre métodos morfológicos, posicionados no espectro de Gauthier \& Gilliand 
(2006), e métodos de desenho generativo formalmente sequenciáveis, como demonstram Duarte et al. (2012), pode ser materializado numa segmentação. Essa proposição, que será demonstrada em diante, classifica o planejamento em três etapas aninhadas numa sequência compatível com a motivação conceitual resultante - uma ontologia comum aos estágios.

\subsection{O módulo cognitivo ou a análise}

O primeiro exercício estabelece o código empregado em todas as etapas. Em síntese, nessa instância, por um lado, estabelecem-se protocolos instrumentais que reproduzem atributos do objeto de estudo e transladam dados em informação interpretável; e, por outro, estruturam formalmente abstrações lógicas, materializado-as em parâmetros métricos, e orientam concepções geradas nas funções internas do sistema.

Ações nesse módulo referem-se à observação dos dados primários. Os resultados dependem de características do instrumento empregado, como sua precisão e adequação ao problema. Dimensões dos objetos de estudo (i.e. redes; territórios), são reproduzidas em espaços topológicos e submetidas a operações (e.g. convoluções; tesselações), para apresentar níveis de agregação que obedeçam ao rigor estatístico almejado e materializem abstrações teóricas, como magnitude e diversidade, em formato paramétrico.

Outro sentido é edificar o código que represente atributos endógenos. A exploração empírica de padrões urbanos pode inspirar modelagens de processos e, por limitações gramaticais, restringir soluções, o que será discutido nos módulos posteriores. Nesse módulo, criar gramáticas, compostas por regras de formatos ou descrições, converge para a descrição empírica, porque a análise pode apenas emular linguagens naturais do contexto, algo aquém do potencial criativo (BEIRÃO, 2012, p. 73). Essa lacuna será administrada nos outros módulos. Pode-se convencionar, então, que esse estágio manifesta um exercício morfológico estritamente cognitivo (GAUTHIER; GILLILAND, 2006).

\subsection{O módulo expressivo ou a modelagem}

Wegener (2004) decompõe o sistema urbano em processos que são simultaneamente influenciados por forças de mercado e por regulação pública. Esses submodelos, demonstrados na Figura 2, dispõem-se num espectro conforme a velocidade das mudanças, com exceção do subsistema ambiental, que interage com impactos dos demais processos endógenos e com processos exógenos complexos.

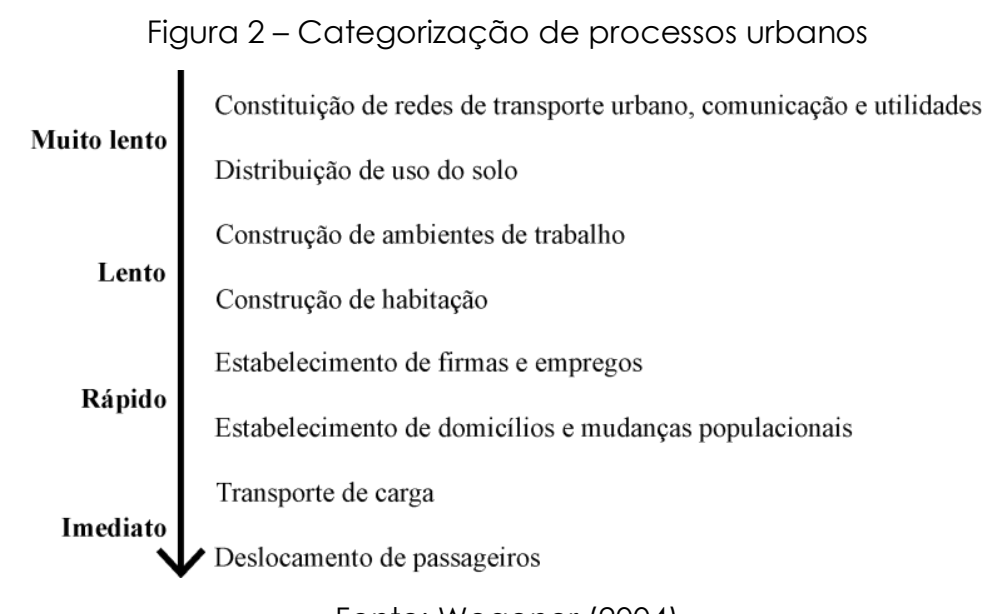

Fonte: Wegener (2004)

Reconhecendo esses processos, as estruturas lógicas que os geram são conceitualmente deliberadas e convertidas a um substrato algébrico, por métodos como a análise de trajetórias. Naturalmente, devem ser coerentes com o módulo cognitivo, que constitui a interface direta com o objeto. Logo, a análise empírica anterior conceitua o módulo 
expressivo por avaliações singulares, descompromissadas com sua estrutura, e, simultaneamente, enquadra-se como instrumento necessário para formalizar e calibrar as emulações conjecturadas.

O desafio de modelar o sistema urbano consiste, então, dos seguintes argumentos: (i) 0 sistema se fundamenta em subsistemas que interagem entre si de forma tão complexa quanto seus mecanismos internos, que são pouco previsíveis; (ii) enquanto a cidade ganha proporção, a organização socioespacial volta-se a nichos geográficos crescentemente intrincados, conforme o número de subconjuntos da rede espaço-temporal e combinações que compõem as relações; (iii) a fragmentação sociodemográfica, que já institui adversidade cognitiva para ser representada, implica ou em processos paralelos, particulares a cada parcela, que determina quão numerosos serão os detalhes, ov em processos divergentes, que subentende competição entre atores por critérios múltiplos. Neste caso, jogos matemáticos são fundados porque objetivos e critérios são individualizados. Na próxima seção, essa dificuldade aumenta quando objetivos do planejador, distintos dos demais sujeitos, são inseridos.

\subsection{O módulo normativo ou a prescrição}

Uma abordagem se torna generativa quando protocolos e prognósticos são transformados em prescrições. Para eficácia do problema, o desenho pode se basear em gramáticas mais criativas do que as puramente empíricas (cf. BEIRÃO, 2012; DUARTE et al., 2012) do módulo cognitivo.

Para fornecer soluções orientadas por objetivos, representados por indicadores produzidos no módulo expressivo, é necessário realizar tanto explorações conceituais - o que são e como representar objetivos - quanto escolhas metodológicas - como soluções são geradas e configuradas no problema. Uma ação busca a literatura para conceber funções-objetivos e métricas de avaliação, outra significa resolver problemas matemáticos para gerar e avaliar soluções.

Soluções podem ser geradas por heurísticas limitadas por gramáticas, simuladas pelo módulo expressivo e avaliadas por funções de desempenho, vide Duarte et al. (2012). Esses procedimentos, mesmo iterativos, não necessariamente produziriam ótimos globais, mesmo concebendo boas soluções. De maneira exploratória, é recomendável conhecer o limite superior do desempenho: torna-se conveniente modelar por programação matemática.

A programação desse problema é caracterizada por jogos. Como comentado, o sistema já é composto por escolhas individuais conflituosas, referentes aos atores dos processos urbanos. Entretanto, quando o objetivo do planejador interdepende com a ação desses sujeitos, outro tipo de jogo é formado. O programa aparelha-se a restrições de equilíbrio, ou seja, um problema binível composto por um jogo não cooperativo e um de liderança-quantidade (FARAHANI et al., 2013).

\subsection{Ontologia programática e orientação epistêmica}

As etapas devem estar explicitamente alinhadas com critérios de avaliação estabelecidos e sua conjuntura teórica. Demonstradas pela Figura 3, relações entre módulos que transformam dados (setas pretas) são francamente dispostas, enquanto relações ontológicas (vermelhas) e teóricas (azuis) se espalham, o que garante certa coesão: os objetivos gerais suscitam a concepção de protocolos endógenos para conduzir preferências metodológicas. 
Figura 3 - Fluxograma de relações metodológicas, ontológicas e teóricas entre módulos

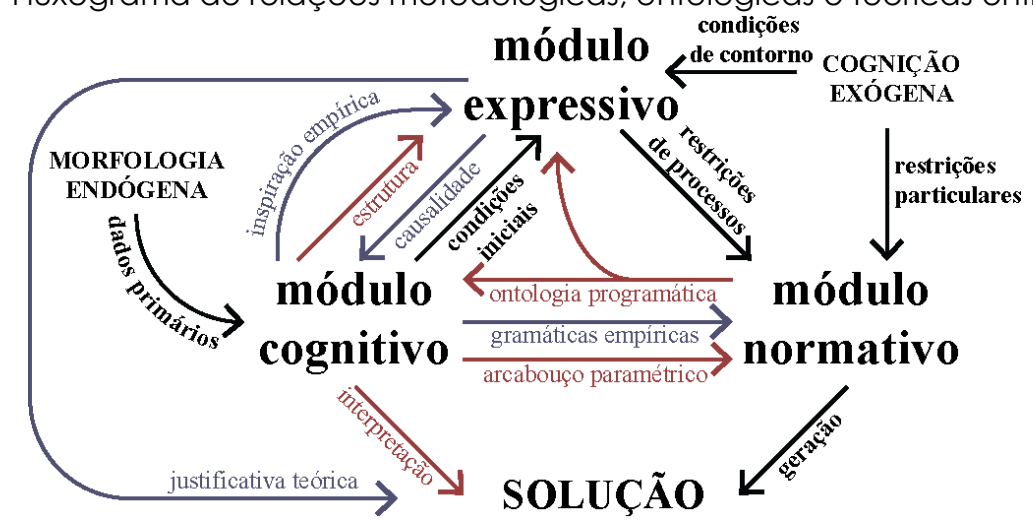

Fonte: $\mathrm{O}$ autor

Em problemáticas que envolvam interação entre transportes e uso do solo, é conveniente voltar-se às dimensões controláveis. Segundo Gauthier \& Gilliland (2006), a classificação entre abordagens epistêmicas depende da natureza dos termos formais - onde (e se) estão dentro do sistema declarado. Logo, abordagens menos externalistas moldariam problemas relacionados às dimensões do sistema - como parâmetros tecnológicos ou urbanísticos, e.g. ocupação de veículos. Portanto, resoluções que partem dessa integração são essencialmente internalistas se sustentadas por condições de contorno em vez de processos paralelos completamente modelados.

\section{CONSIDERAÇÕES FINAIS}

A concepção desse método generativo resultou em módulos que transformam dados primários em soluções metodicamente orientadas a objetivos, reconhecedoras de processos urbanos. Pela inclinação internalista, sinaliza-se que apenas representantes implicitamente relacionados, por teoria, ou impactos aproximados seriam empregados em funções-objetivo fora do escopo setorial (eg. saúde ou produtividade). Entretanto, atributos do sistema integrado apresentam dimensões fundamentalmente socioeconômicas. Agentes individuais formam composições que poderiam ser exploradas em objetivos que envolvam distribuição espacial e/ou diversidade demográfica. Assim, a justiça socioespacial pode ser endogenamente investigada, mesmo não explorado todo o campo socioeconômico.

Por fim, resta qualificar quanto sistemas exógenos afetam processos de mudança urbana. Diferente do transporte, o uso do solo modifica-se lentamente. Isso implica que são adequados em matemática singela em vez de contínua e que erros da modelagem se acumularão mais definitivamente. Na programação, já complicada, fatores estocásticos (ou aleatórios!) dos processos modificam o impacto das decisões. Isso deve ser continuado com mais pesquisa.

\section{REFERÊNCIAS}

BEIRÃO, J. N. Clty Maker / Designing Grammars for Urban Design. Delft: Delft University of Technology, 2012.

DUARTE et al. City Induction: A Model for Formulating, Generating, and Evaluating Urban Designs. In: STEFAN MÜLLER ARISONA et al. (Eds.). . Digital Urban Modeling and Simulation. Berlin: Springer, 2012. p. 73-98.

FARAHANI, R. Z. et al. A review of urban transportation network design problems. European Journal of Operational Research, v. 229, n. 2, p. 281-302, 2013.

FISCHER, T.; HERR, C. M. Teaching Generative Design. [s.l: s.n.]. 
GAUTHIER, P.; GILLILAND, J. Mapping urban morphology: a classification scheme for interpreting contributions to the study of urban form. Urban Morphology, v. 10, n. 1, p. 41-50, 2006.

KAMRUZZAMAN, M. et al. Advance transit oriented development typology: Case study in brisbane, australia. Journal of Transport Geography, v. 34, p. 54-70, 2014.

LIMA, F.; KÓS, J. R. Pensamento algorítmico, parametrização e urbanismo sustentável: uma avaliação de parâmetros para estratégias de projeto urbano inteligente. Proceedings of the XVIII Conference of the Iberoamerican Society of Digital Graphics - SIGraDi: Design in Freedom. Anais...São Paulo: Editora Edgard Blücher, dez. 2014Disponível em:

<http://www.proceedings.blucher.com.br/article-details/14289>

LIMA, F. T. DE A. Métricas Urbanas: Sistema (para)métrico para análise e otimização de configurações urbanas de acordo com métricas de avaliação de desempenho. Rio de Janeiro: Universidade Federal do Rio de Janeiro, 2017.

LYU, G.; BERTOLINI, L.; PFEFFER, K. Developing a TOD typology for Beijing metro station areas. Journal of Transport Geography, v. 55, p. 40-50, 2016.

PICON, A. Algorithmic Architecture or the Computer as a Double? In: KOSTAS TERZIDIS (Ed.). Algorithmic Architecture. [s.l.] Routledge, 2006. p. vii-x.

WEGENER, M. Overview of Land Use Transport Models. In: HENSHER, D. A. et al. (Eds.). Handbook of Transport Geography and Spatial Systems. Handbooks in Transport. Bingley: Emerald Insight, 2004. v. 5p. 127-146.

ZEMP, S. et al. Classifying railway stations for strategic transport and land use planning: Context matters! Journal of Transport Geography, v. 19, n. 4, p. 670-679, 2011. 